\title{
An extended target particle probability hypothesis density filter based on the Star-Convex shape estimation
}

\author{
Zhuo $\mathrm{Cao}^{1, \mathrm{a}}$, Xinxi Feng ${ }^{1, \mathrm{~b}}$, Lei $\mathrm{Pu}^{1, \mathrm{c}}$ \\ ${ }^{1}$ Information and Navigation College of Air Force Engineering University, Xi'an, 710077, China \\ aemail: 328988073@qq.com, bemail: fengxinxi2005@aliyun.com, cemail: 304019416@qq.com
}

Keywords: Information Fusion; Target Tracking; Track Initiation; Measurement Partition

\begin{abstract}
The extended target is characterized by common centroid kinematic state and extended information, extended forms not only can be treated as a state to be estimated separately, including the size, shape and direction information will effectively enhance the performance of filter with proper use. For this reason, a new algorithm of the extended target particle probability hypothesis density filter modeling for Star-Convex is proposed, the algorithm take local clustering trend analysis into account and propose a method of extended target track initiation based on Star-Convex gate, then, according to the different characteristics of measurement sets, we propose an adaptive measurement partition algorithm based on extended information of Star-Convex. Simulation results show that the false initiation and computational cost both reduce significantly. In the intersection or the neighbor target tracking scenario, the proposed partition algorithm can maintain a better performance and improve the stability of the filter.
\end{abstract}

\section{Introduction}

In traditional target tracking, the extended shape information is negligible compared with the resolution of sensor, and the filter is estimated based on the single point measurement [1]. However, with the increase of sensor technology, several reflection points can be received from the target surface at every moment, even if the limited resolution make these points distinguished partly[2], a number of measuring points provide shape information more than kinematic state, this type of target calls extended target. The extended target tracking problem was first proposed by Drummond[3], which attracted much attention and extensive research. In 2005, Gilholm[4] proposed the method of spatial probability distribution to establish the target model, which assumes that the number of extended target measurements and clutter measurements obey Poisson distribution, but the model doesn't consider the shape information. Since then, Gilholm[5] proposed to model with ellipse. However, the estimation of the shape information is at the level of parameter estimation, which leads to a larger fluctuation in the shape estimation results. Later Koch[6] introduced random positive definite matrix to model the extended form, however, the symmetric positive definite matrix can only describe an ellipsoidal target, it is difficult to characterize the abundant shape of movement targets, so reference[7] presents a method of modeling with Star-Convex extended form based on Random Hypersurface Model(RHM).

This paper proposes an extended target particle probability hypothesis density filter with shape modeling for Star-Convex, which is a kind of nonlinear filter and it can estimate kinematic state and extended form with irregular shape accurately. According to introducing local clustering trend analysis theory to the extended target track initiation, we use the extended target shape information and propose a new initiation algorithm based on Convex-Star gate, which significantly reduces the probability of false initiation and improve the efficiency of the filter. Then it proposes an adaptive measurement partition algorithm based on Star-convex shape estimation, which can effectively estimate the number and kinematic state of extended target even on the condition that intersection or the neighbor target tracking scenario. 


\section{Extended Target Particle Probability Hypothesis Density Filter Based on Star-Convex}

The shape and size of the target are abundant, thus the conventional triangle and ellipse are difficult to model the precise details of the extended target. The Star-Convex outline can be adjusted arbitrarily by fitting the parameters. Extended target PHD can be realized by Gauss mixture or particle filter. However, Gauss mixture is based on the assumption that the state noise and measurement noise obeys Gauss distribution, thus it doesn't apply to non Gauss nonlinear tracking system. This paper combines the particle PHD and Star-Convex measurement modeling so as to represent the kinematic state and extended shape information.

\section{(1)Star-Convex Outline Modeling}

This paper assumes the modeling of Star-Convex extended target in two-dimensional plane:

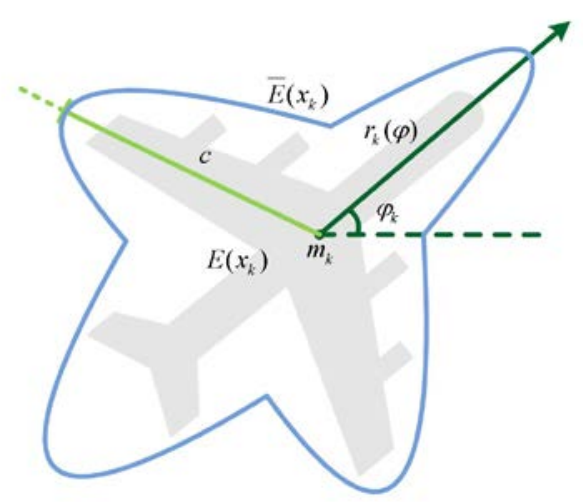

Fig.1. The diagram of Star-Convex

As shown in Figure 1, extended target measurement distribute at the boundary which the centroid is $m_{k}, \bar{E}\left(x_{k}\right)$ is used to represent the boundary curve, $E\left(x_{k}\right)$ represent all the measurement points in $\bar{E}\left(x_{k}\right), r_{k}(\varphi)$ represent the line between centroid and the boundary.

Fourier series function of $r_{k}(\varphi)$ can be expressed as:

$$
r_{k}(\varphi)=\frac{a_{k}^{0}}{2}+\sum_{i=1}^{N} a_{k}^{n} \cos (n \varphi)+b_{k}^{n} \sin (n \varphi)
$$

The location of the extended target surface measurement is called the measurement source [8], the measurement data is generated by the measurement source, and the number and position of measurement source will change in different time. The measurement source model of Star-C onvex using RHM can be represented as:

$$
y_{k}=m_{k}+s_{k} \cdot r_{k}(\varphi) \cdot[\cos (\varphi), \sin (\varphi)]^{T}
$$

In the formula, the position of centroid is expressed as $m_{k}$, and the scale control factor is expressed as $s_{k}$, and the scale is $[0,1]$, which cover all the reflection points on the target surface. $[\cos (\varphi), \sin (\varphi)]^{T}$ is the unit vector with angle of $\varphi$. The measurement $z_{k}$ generate from measurement source, which can be represented as:

$z_{k}=y_{k}+v_{k}$

The nonlinear measurement equation can be obtained by formula (2) and (3):

$z_{k}=m_{k}+s_{k} \cdot r_{k}(\varphi) \cdot[\cos (\varphi), \sin (\varphi)]^{T}+v_{k}=h\left(x_{k}, s\right)+v_{k}$

\section{(2)Extended Target Particle Probability Hypothesis Density Filter}

Extended target particle PHD filter regard target state and measurement as random finite sets.

$$
\begin{aligned}
& \boldsymbol{X}_{k}=\left\{\boldsymbol{x}_{k}^{1}, \boldsymbol{x}_{k}^{2}, \ldots, \boldsymbol{x}_{k}^{N_{x, k}}\right\} \\
& \boldsymbol{Z}_{k}=\left\{\mathbf{z}_{k}^{1}, \mathbf{z}_{k}^{2}, \ldots, \mathbf{z}_{k}^{N_{z, k}}\right\}
\end{aligned}
$$

In the formula, the elements of target state and measurement are disordered in finite sets.

It is assumed that all targets and measurement are independent of each other, and the number of 
extended targets and clutter measurements yields Poisson distribution.

The measurement update PHD can be expressed as:

$D_{k \mid k}(\boldsymbol{x} \mid \mathbf{z})=L_{Z}(\boldsymbol{x}) D_{k \mid k-1}(\boldsymbol{x} \mid \mathbf{z})$

In the formula, the likelihood function is in the expression of $L_{Z}(x)$ :

$$
L_{Z}(\boldsymbol{x}) \triangleq 1-\left(1-\mathrm{e}^{-\gamma(\boldsymbol{x})}\right) P_{D}(\boldsymbol{x}) D_{k \mid k-1}(\boldsymbol{x} \mid \mathbf{z})+\mathrm{e}^{-\gamma(\boldsymbol{x})} P_{D}(\boldsymbol{x}) \sum_{\boldsymbol{P} \angle \mathbf{Z}_{k}} \omega_{P} \sum_{W \in \boldsymbol{P}} \frac{\gamma(\boldsymbol{x})^{|\boldsymbol{W}|}}{d w} \cdot \prod_{Z_{k} \in W} \frac{\phi_{z}(\boldsymbol{x})}{\lambda_{k} C_{k}\left(\mathbf{z}_{k}\right)}
$$

By means of formula (4), the closed form solution of likelihood function $\phi_{z}(x)$ for describing single Star-Convex is expressed as:

$$
\phi_{z}(\boldsymbol{x})=f\left(z_{k} \mid x_{k}\right)=\iint \delta\left[z_{k}-\left(h\left(x_{k}, s\right)+v_{k}\right)\right] \cdot N\left(v_{k} ; 0, R_{k}\right) \cdot f^{s}(s) d v_{k} d s=\int N\left[z_{k}-h\left(x_{k}, s\right) ; 0, R_{k}\right] \cdot f^{s}(s) d s
$$

$f^{s}(s)$ is the distribution function of scale control factor $s$.

In order to facilitate mathematical processing, the log-likelihood function is expressed as :

$$
\log \left(f\left(Z_{k} \mid x_{k}\right)\right)=\sum_{i=1}^{N_{z, k}} \log f\left(z_{k}^{i} \mid x_{k}\right)
$$

\section{Multiple extended target track initiation}

When tracking extended target using random finite set theory, track initiation problem is not only the accurate establishment of track start, but also provide the initial value for the PHD filter, so the track initiation greatly affect the overall performance of the filter. The existing method of extended target track initiation is similar to the initiation of center-group target, which extract the centroid of the extended target and represent the kinematic state of the extended target.

When cluttering to the measurement from the cluster environment implies the predetermined assumptions that the measured data can be divided, therefore, the spatial clustering operation can get a clustering result, Which may result in two consequences, one is if the measurement data are random, the clustering results can't be explained; the other is the lack of a priori knowledge about the measurement set will bring great difficulties to the selection of algorithms and parameters.

All the measurements consist of target generation measurement and spatial clutter measurement. Spatial clutter usually caused by the interference of adjacent target, electromagnetic and meteorological, thus they are usually random in quantity, density and spatial distribution. So it can be considered that the target generation measurements are the main source of poly. In order to reduce the false clustering framework imposed on the random distribution,it's necessary to make cluster tendency before clustering.

All the measurements consist of target generation measurement and spatial clutter measurement. Spatial clutter usually caused by the interference of adjacent target, electromagnetic and meteorological, thus they are usually random in quantity, density and spatial distribution. So it can be considered that the target generation measurements are the main source of poly. In order to reduce the false clustering framework imposed on the random distribution,it's necessary to make cluster tendency before clustering. 


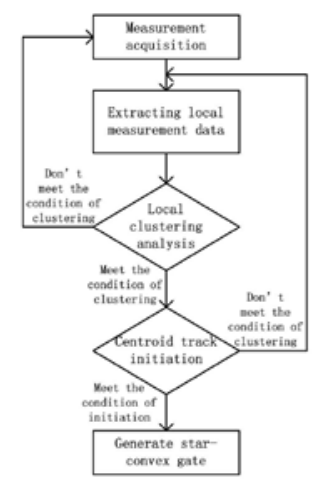

Fig.2. The diagram of multiple extended target track initiation

In summary, this paper analysis the local clustering tendency based on Star-Convex prediction gate at extended target track initiation. The implementation process is shown in Figure 2.

In this paper, we take the Nearest Neighbor Index (NNI) method to analyze the clustering trend of the measurement [8]. The nearest neighbor index NNI is expressed as

$$
N N I=\frac{d(N N)}{d(\text { ran })}=\frac{\sum_{i=1}^{N} d_{i}^{\text {min }} / N}{d(\text { ran })}
$$

$d_{i}^{\text {min }}$ represents the nearest neighbor measurement distance; $N$ represents the number of measurement; $d$ (ran)represents the average nearest neighbor distance of random distribution and we set it as $d($ ran $)=0.5 \sqrt{A / N}$, When $N N I<1$, the measurement should be an aggregation distribution, When $N N I=1$, the measurement should be a random distribution, When $N N I>1$, the measurement should be an uniform distribution.

\section{Multiple Extended Target Measurement Partition}

In this paper, based on the OPTICS partition and prediction partition with Star-Convex shape estimation, we propose an adaptive measurement set partition method. For solving the problem of filter performance decreasing in the intersection or the neighbor target tracking scenario, we proposes a local measurement partition strategy.

In track initiation stage, for the track prediction information is not complete, the estimation accuracy is poor, so we use OPTICS method in this condition, and the OPTICS algorithm is a kind of density based algorithm. The algorithm is not sensitive to noise and adopt density ordering strategy, which establish the concept of core distance and reached distance to find arbitrary shape and different density extended target measurement. However the OPTICS algorithm is still a kind of partition based on density which could cause filter performance decreasing in the intersection or the neighbor target tracking scenario.

Therefore, after the track initiation, the prediction partition based on Star-Convex shape is adopted. $\hat{x}_{k \mid k-1}^{j}$ is the state prediction of extended target $j$ at time $k$, the angle between state prediction and measurement $z_{i}$ is expressed as $\varphi_{i}$. When the measurements meet the condition of formula (12), they are divided into the same cluster.

$$
\begin{aligned}
& {\left[z_{i}-h\left(\hat{x}_{k \mid k-1}^{j}, s\right)\right]^{T} \cdot\left[z_{i}-h\left(\hat{x}_{k \mid k-1}^{j}, s\right)\right]<\varepsilon\left(\varphi_{i}\right)} \\
& \varepsilon\left(\varphi_{i}\right)=\lambda r_{k}\left(\varphi_{i}\right)
\end{aligned}
$$

The traditional measurement partition can only partition the measurements to a specific extended target, it is simple, however in the intersection or the neighbor target tracking scenario, it's difficult to partition accurately. Here, when the measurements of target 1 and target 2 overlap, we make full use of the prediction Star-Convex shape prediction to restrict the range of actual position occurring intersection, after then, we do the re-partition to the measurements in the range of intersection and 
put them into several cluster so as to make up the impact caused by the sensor cannot receive measurements from different overlapping targets at the same time.

\section{Simulation Experiment and Result Analysis}

We assume the extended targets with Star-Convex shape, the observation area $\mathrm{x} \sim[-6000 \mathrm{~m}$, $10000 \mathrm{~m}], \mathrm{y} \sim[-10000 \mathrm{~m}, 10000 \mathrm{~m}]$, the sampling period is $T=1 \mathrm{~s}$, the survival probability is $P_{s}=0.99$, the detection probability is $P_{s}=0.99$. To verify the performance of the proposed algorithm, two experimental scenarios are simulated with Monte Carlo 200 times.

\section{Simulation experiment scenario 1}

In order to fully verify the initiation performance of the proposed algorithm, the experiment compare the proposed initiation algorithm with the algorithm proposed by reference [9], as shown in Table 1, four moving targets appear one by one, the motion parameters set as follows.

Table 1 Target motion parameter setting diagram

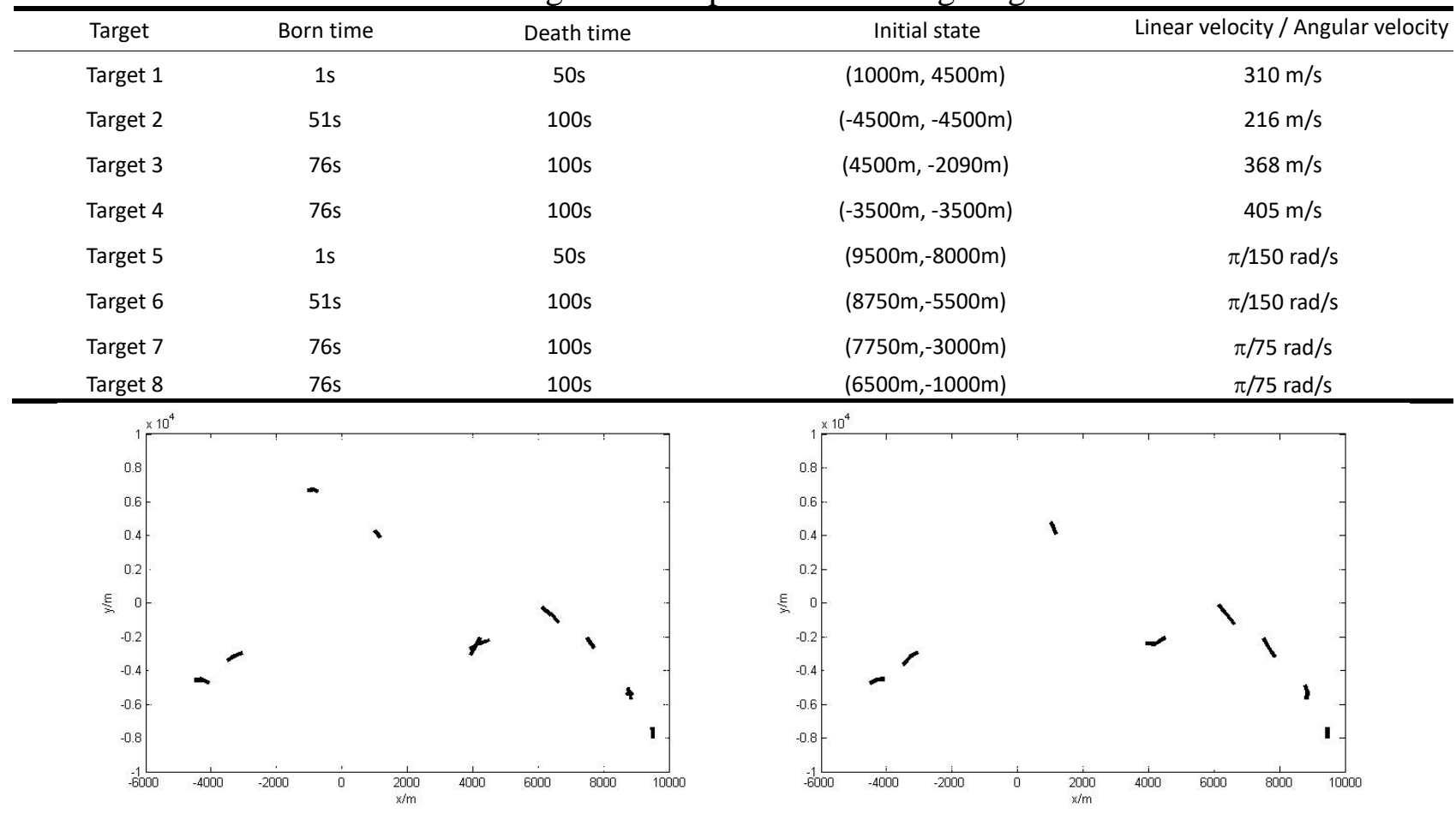

Fig.3 The track initiation diagram of extended target centroid based on 2/3 logic

Fig. 4 The track initiation diagram of local clustering tendency analysis based on Star-Convex gate

Figure 3 shows the initiation track based on the 2/3 logical center extended target initiation method. The initiation track generated by the proposed algorithm is shown in Figure 4. We can conclude that the method of proposed algorithm based on local clustering analysis using Star-Convex shape estimation is much better than center extended target initiation algorithm.

$$
q_{t f}=\frac{p_{f}+\frac{T_{\text {ture }}-T_{\text {initiation }}}{T_{\text {ture }}}}{p_{t}}
$$

In the formula, $T_{\text {ture }}$ represent the true track number, $T_{\text {initiation }}$ represent the number of initiation track. $q_{t f}$ represent the comprehensive performance index. We can conclude that the small value of $q_{t f}$ represent a good initiation performance. 
Table 2 Comparison of track initiation performance

\begin{tabular}{ccccccc}
\hline \multirow{2}{*}{$\begin{array}{c}\text { Number of } \\
\text { clutter }(\lambda)\end{array}$} & \multicolumn{2}{c}{$\begin{array}{c}\text { Correct initial probability } \\
\left(p_{t}\right)\end{array}$} & $\begin{array}{c}\text { False initial probability } \\
\left(p_{f}\right)\end{array}$ & \multicolumn{2}{c}{$\begin{array}{c}\text { Initial performance index } \\
\left(q_{f}\right)\end{array}$} \\
\cline { 2 - 7 } & The proposed & $\begin{array}{c}\text { The logical } \\
\text { algorithm }\end{array}$ & $\begin{array}{c}\text { The proposed } \\
\text { algorithm }\end{array}$ & $\begin{array}{c}\text { The logical } \\
\text { algorithm }\end{array}$ & $\begin{array}{c}\text { The proposed } \\
\text { algorithm }\end{array}$ & $\begin{array}{c}\text { The logical } \\
\text { algorithm }\end{array}$ \\
\hline 20 & $100 \%$ & $100 \%$ & $0.18 \%$ & $1.64 \%$ & 0.0018 & 0.0164 \\
40 & $99.33 \%$ & $99.28 \%$ & $0.27 \%$ & $3.45 \%$ & 0.0094 & 0.0416 \\
60 & $97.45 \%$ & $97.80 \%$ & $0.48 \%$ & $6.04 \%$ & 0.0843 \\
80 & $93.68 \%$ & $94.27 \%$ & $1.34 \%$ & $15.15 \%$ & 0.0818 & 0.2245 \\
\hline
\end{tabular}

As can be seen from Table 2, with the increasing number of clutter measurements, both two algorithms can maintain a high correct initial probability. The main difference between two algorithms is the proposed algorithm in the clutter environment can still maintain a low level of false initiation probability, this is because through the local clustering trend analysis based on the Star-Convex, effectively reduce the false clustering framework imposed on the measurements of random distribution, which avoid the appearance of unaccountable equivalent measurements, so that the false initiation probability can decrease.

\section{Simulation experiment scenario 2}

The experiment compare the proposed partition algorithm with the Distance partition algorithm and OPTICS partition algorithm. As shown in Table 3, the motion parameters set as follows.

Table 3 Target motion parameter setting diagram

\begin{tabular}{ccccc}
\hline Target & Born time & Death time & Initial position & Line velocity/Angular velocity \\
\hline Target 1 & $1 \mathrm{~s}$ & $50 \mathrm{~s}$ & $(-4000 \mathrm{~m}, 4000 \mathrm{~m})$ & $253 \mathrm{~m} / \mathrm{s}$ \\
Target 2 & $1 \mathrm{~s}$ & $50 \mathrm{~s}$ & $(-4000 \mathrm{~m}, 0 \mathrm{~m})$ & $253 \mathrm{~m} / \mathrm{s}$ \\
Target 3 & $1 \mathrm{~s}$ & $50 \mathrm{~s}$ & $(-5125 \mathrm{~m},-6250 \mathrm{~m})$ & $2 \pi / 375 \mathrm{rad} / \mathrm{s}$ \\
Target 4 & $1 \mathrm{~s}$ & $50 \mathrm{~s}$ & $(-5375 \mathrm{~m},-6500 \mathrm{~m})$ & $2 \pi / 375 \mathrm{rad} / \mathrm{s}$ \\
\hline
\end{tabular}

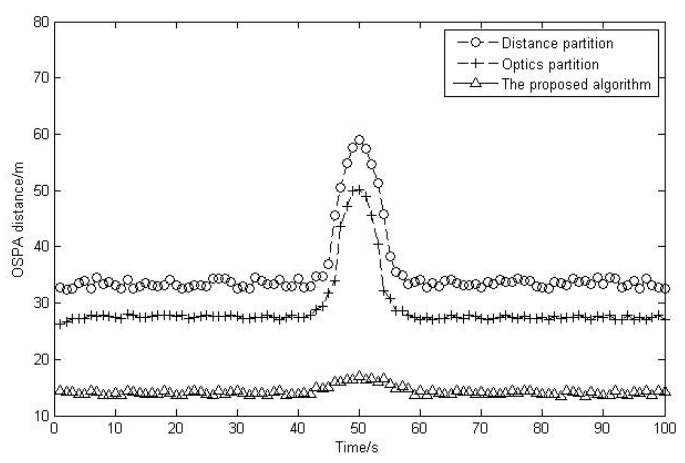

Fig. 5 The diagram of OSPA distance

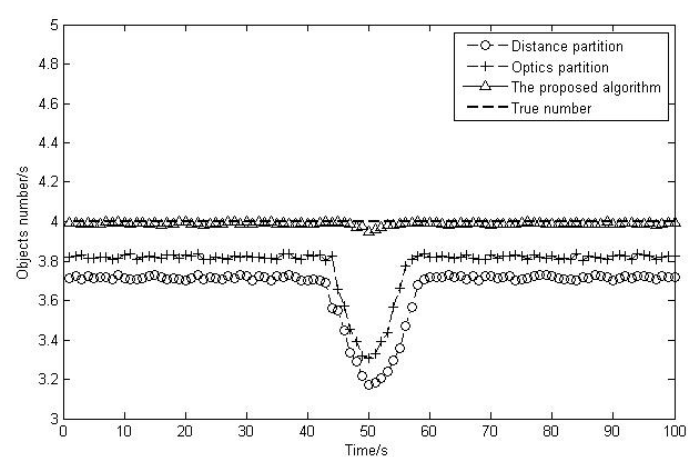

Fig. 6The diagram of target number estimating

It can be seen from above that the partition accuracy of the proposed algorithm is significantly better than the other two algorithms, and the two traditional algorithm lead to the target number estimation underestimated.

\section{References}

[1] Shape estimation of extended targets based on Gaussian surface feature matrix[J]. Journal of Optoelectronics •Laser, 2014, 25(9):1803-1811.

[2] Liu M Q, Lan J. Advance theory and application of target tracking[M]. Beijing: Science Press, 2015: 202-203. [3] Zhizhong Yin. Application of FPGA control DC motor servo system [J]. Inner Mongolia Science and Technology and Economy, 2008177 (23) 101-103.

[3] Drummond O E, Blackman S S, Petrisor G C. Tracking cluster and extended objects with multiple sensors[C]. Proc. of the SPIE International Conference on Signal and Data Processing of 
Small Targets, 1990: 362-371.

[4] Gilhilm K, Salmond D. Spatial distribution model for tracking extended objects[J]. IEE Proceedings on radar, sonar and navigation, 2005, 152(5): 364-371.

[5] Gilhilm K, Godsill S,Salmond D. Poisson models for extended target and group tracking[C]. Proceedings of the data processing of small targets. San Diego, CA: SPIE, 2005: 230-241.

[6] Koch J W. Bayesian approach to extended objects and cluster tracking using random matrices[J]. IEEE Transactions on aerospace and electronic systems, 2008, 44(3): 1042-1059.

[7] Han Y L, Zhu H Y, Han C Z, Wang J. Gaussian-mixture probability hypothesis density filter for multiple extended targets[J]. Journal of Xi'an JiaoTong University, 2014, 48(4): 95-104.2010 (5) 86-87.

[8] Deng M, Liu Q L, Li G Q, et al. Spatial clustering analysis and its application[M]. Beijing: Science Press, 2011: 43-49.

[9] Lan J, Li X R. Tracking of maneuvering Non-Ellipsoidal extended object or target group using random matrix[J]. IEEE Transactions on Signal Processing, 2014, 62(9): 2450-2463. 